\title{
INTERPOLATION BY SERIES OF EXPONENTIALS IN $H(D)$ WITH REAL NODES
}

\section{S.G. MERZLYAKOV, S.V. POPENOV}

\begin{abstract}
In the space of holomorphic functions in a convex domain, we study a problem on interpolation by sums of the series of exponentials converging uniformly on compact subsets of the domain. The discrete set of multiple interpolation nodes is located on the real axis in the domain and has the unique finite accumulation point. We obtain a solvability criterion in terms of distribution of limit directions at infinity for the exponents of exponentials.
\end{abstract}

Keywords: holomorphic function, convex domain, interpolation with multiplicities, series of exponentials, closed ideal, closed submodule, strong dual space, duality

Mathematics Subject Classification: 30E05

\section{Formulation of THE PROBLEM AND PRELIMINARIES}

Let $D$ be a convex domain in $\mathbb{C}$. We denote by $H(D)$ the space of holomorphic functions in $D$ with the topology of uniform convergence on compact sets in $D$. We consider an arbitrary discrete set of complex numbers $\Lambda=\left\{\lambda_{n}\right\}_{n \in \mathbb{N}}$ in $\mathbb{C}$.

We denote

$$
\Sigma(\Lambda, D)=\left\{f \in H(D): f(z)=\sum_{n=1}^{\infty} c_{n} e^{\lambda_{n} z}, z \in D\right\} .
$$

The convergence of the series of exponentials is supposed to be absolute for each point $z \in$ $D$, then ([1]) such series converges in the topology of space $H(D)$. For a multi-dimensional situations it was shown, for instance, in work [2].

Suppose that set $D \cap \mathbb{R}$ is non-empty. We assume that in $D$ we are given an infinite discrete set real interpolation nodes $\mathcal{M}=\left\{\mu_{k}\right\}_{k=1}^{\infty}, \mathcal{M} \subset D \cap \mathbb{R}$. We also assume that to each node $\mu_{k} \in \mathcal{M}$ a multiplicity $m_{k} \in \mathbb{N}$ is associated. If $f, g \in H(D)$, we shall write $f \cong g$ on $\mathcal{M}$, if $f^{(j)}\left(\mu_{k}\right)=g^{(j)}\left(\mu_{k}\right)$ for each $k \in \mathbb{N}$ and $j=0,1, \ldots, m_{k}-1$.

In $H(D)$ we consider the following problem on interpolation by means of series of exponentials with real nodes:

For an arbitrary set of nodes $\mathcal{M} \subset D \cap \mathbb{R}$ and for each function $g \in H(D)$ there exists a function $f \in \Sigma(\Lambda, D)$, such that $f \cong g$ on $\mathcal{M}$.

By the classical result of interpolation by holomorphic functions [3, Corol. 1.5.4], this problem can be formulated in terms of traditional notations:

For each interpolation data $b_{k}^{j} \in \mathbb{C}, k \in \mathbb{N}, j=0,1, \ldots, m_{k}-1$, there exists a function $f \in$ $\Sigma(\Lambda, D)$, such that $f^{(j)}\left(\mu_{k}\right)=b_{k}^{j}$, for each $k$ and $j$.

S.G. Merzlyakov, S.V. Popenov, Interpolation by series of exponentials in $H(D)$ With Real NODES.

(c) Merzlyakov S.G., Popenov S.V. 2015.

The work was supported by RFBR (grant 11-01-00572-a).

Submitted October 27, 2014. 
We denote by $\psi_{\mathcal{M}}$ the function in $H(D)$ with zeroes at all the nodes $\mu_{k} \in \mathcal{M}$, with multiplicities $m_{k}$, and only at these nodes. We denote by

$$
\left(\psi_{\mathcal{M}}\right)=\left\{h \in H(D): h=\psi_{\mathcal{M}} \cdot r, r \in H(D)\right\}
$$

the closed ideal in $H(D)$ generated by function $\psi_{\mathcal{M}}$. It is easy to see that $\left(\psi_{\mathcal{M}}\right)=I_{\mathcal{M}}=\{h \in$ $H(D): h \cong 0$ on $\mathcal{M}\}$.

Given a set of nodes $\mathcal{M}$, the solvability of problem on interpolation in $H(D)$ by the sums of series of exponentials with the exponents in a prescribed set $\Lambda$ is equivalent to the validity of the following representation:

$$
H(D)=\Sigma(\Lambda, D)+\left(\psi_{\mathcal{M}}\right)
$$

Under the conditions of the considered problems, the interpolation can be non-unique, i.e., $\Sigma(\Lambda, D) \cap\left(\psi_{\mathcal{M}}\right) \neq\{0\}$. It was proven in work [4] for the space of entire functions and the proof adduced in this work can be adapted for our case with obvious changes.

If representation (2) holds true and $\Sigma(\Lambda, D) \subset X \subset H(D)$, the representation $H(D)=X+$ $\left(\psi_{\mathcal{M}}\right)$ is valid.

In the case $D=\mathbb{C}$ and $X$ is the kernel of some convolution operator in the space of entire functions $H(\mathbb{C})$, in work [5] there were found sufficient conditions for interpolation of functions in the kernel of the convolution operator in terms of location of zeroes $\Lambda$ for the characteristic function of this operator. In [5] set $\mathcal{M}$ had two accumulation points $\pm \infty$. In work [4] we succeeded to find other ways of proving and for all possible cases of location of accumulation points for $\mathcal{M}$ we obtained the solvability criterions for the problem on multiple interpolation in $H(\mathbb{C})$ by the series of exponentials in $\Sigma(\Lambda, \mathbb{C}) \subset X$. In the case when the set of nodes had two accumulation points $\pm \infty$, the criterion in [4] was formulated via the same notions as in work [5].

In the present paper the method of proof of sufficiency [4] is extended for the case of holomorphic functions in a convex domain. We obtain the criterion of interpolation in the case when $\mathcal{M}$ has the unique accumulation point, which lies on boundary $\partial D$ of domain $D$. The criterion relates the distribution of limiting directions of exponents in $\Lambda$ at infinity with the geometric structure of the part of convex domain containing this limiting point.

The proof of sufficiency is reduced to interpolation by exponential series in the space of functions holomorphic in some half-plane. Moreover, the proof of necessity in the considered case of one accumulation point is happened to require an idea of completely different nature in comparison with space $H(\mathbb{C})$. The matter is that the series of exponentials converging absolutely on some set possess the property of extending the convergence [2]. It should be noted that the analytic continuation for the elements of common invariant subspaces admitting spectral synthesis was studied in [6].

Remark after the proof. The problem of interpolation in the kernel of convolution operator in a convex domain was considered in work [7].

\section{SCHEME OF REDUCTION TO INTERPOLATION IN THE KERNEL OF CONVOLUTION OPERATOR. DUAL FORMULATION OF INTERPOLATION PROBLEM}

As in work [4], in what follows we employ the scheme of the proof described in work [8]. It is based on the duality with employing the Laplace transform $\mathcal{L}$ of functionals. In the proof of the sufficiency of interpolation conditions, it is proposed to consider natural dual statements independently for each possible location of accumulation points of set $\mathcal{M}$.

Let us describe briefly this scheme; in work [4] it was exposed quite in details for space $H(\mathbb{C})$. For space $H(D)$ we shall indicate some changes required in the arguments of the above cited work. 
We denote by $P_{D}$ the space of entire functions of exponential type with the traditional topology of inductive limit that ensures a topological isomorphism between a strongly dual space $H^{*}(D)$ and space $P_{D}$ obtained by means of Laplace transform $\mathcal{L}$ of functionals $F \in$ $H^{*}(D)$. More precisely, a linear continuous one-to-one Laplace transformation $\mathcal{L}$ of functionals $F \in H^{*}(D)$ is introduced as follows: $\mathcal{L}: F \longmapsto \mathcal{L} F(z)=\left\langle F_{\lambda}, e^{\lambda z}\right\rangle, \mathcal{L} F \in P_{D}$.

The topology in $\left(L N^{*}\right)$-space $P_{D}$ is not described in terms of sequences convergence, however, sequentially closed subspaces happen to be closed ([9]). The exact definition of sequence convergence in this topology will be provided in the proof of sufficiency for Lemma 4.

We introduce a continuous bilinear form $[\cdot, \cdot]: H(D) \times P_{D} \longmapsto \mathbb{C}$ by the formula $[\psi, \varphi]=$ $\left\langle\mathcal{L}^{-1} \varphi, \psi\right\rangle, \psi \in H(D), \varphi \in P_{D}$. By the mapping $\varphi \longmapsto[\cdot, \phi]=\left\langle\mathcal{L}^{-1} \varphi, \cdot\right\rangle$, where $\mathcal{L}^{-1} \varphi \in$ $H^{*}(D)$, defines an isormophism between $P_{D}$ and strongly dual space $H^{*}(D)$. In accordance with introduced duality, each function in space $P_{D}$ is one-to-one correspondence with a linear continuous functional in $H^{*}(D)$.

It is well-known that each function $G \in P_{D}, G \not \equiv 0$, having the minimal type at order one generates in space $H(D)$ the convolution operator $M_{G}: H(D) \longmapsto H(D)$. In the considered duality, this operator can be defined as

$$
M_{G}[\psi](z)=\left[S_{z}(\psi(\lambda)), G_{\lambda}\right]=\left\langle\left(\mathcal{L}^{-1} G\right)_{\lambda}, \psi(z+\lambda)\right\rangle
$$

where $S_{z}$ is the shift operator: $S_{z}(\psi(\lambda))=\psi(\lambda+z)$.

It is known that $M_{G}$ is a linear continuous and surjective operator. The adjoint operator for $M_{G}$ is operator $A_{G}$ of multiplication by characteristic function $G$. It is well-defined on functions $\omega \in P_{D}$ and reads as: $\omega \longmapsto G \cdot \omega$ (for details see [10], [11]).

We denote by $\operatorname{Ker} M_{G}=\left\{f \in H(D): M_{G}[f]=0\right\}$ the kernel of convolution operator $M_{G}$. This kernel is a closed subspace in $H(D)$ invariant w.r.t. the differentiation operator.

Subspace $\operatorname{Ker} M_{G}$ admits spectral synthesis [11], [12], i.e., in the sense of the topology in space $H(D)$ it coincides with the closure of a linear span of the set of all polynomial-exponential monomial $z^{\nu} e^{\lambda_{n} z}$ contained in $\operatorname{Ker} M_{G}$.

Generally speaking, the subspace of series of exponentials $\Sigma(\Lambda, D)$ is not closed in $H(D)$. Because of this fact, in the proof of sufficiency of interpolation conditions, for each possible location of nodes $\mathcal{M}$, we choose a subsequence $\widetilde{\Lambda}$ in $\Lambda$ so that it is the zero set of some entire function $G \in P_{D}$ of minimal type, at that, $\operatorname{Ker} M_{G}=\Sigma(\widetilde{\Lambda}, D)$. Then we prove the validity of representation (2) with $\Lambda$ replaced by $\widetilde{\Lambda}$. But in this case it is also true for $\Lambda$.

Once subsequence $\widetilde{\Lambda}$ is chosen, it is sufficient to show the following two statements.

(I) Subspace Ker $M_{G}+\left(\psi_{\mathcal{M}}\right)$ is everywhere dense in $H(D)$;

(II) Subspace Ker $M_{G}+\left(\psi_{\mathcal{M}}\right)$ is closed in space $H(D)$.

Closed ideal $\left(\psi_{\mathcal{M}}\right)$ was introduced above in $(1)$. Hereafter in this section, to simplify the notations we shall write $\psi=\psi_{\mathcal{M}}$.

If $X_{1}$ is a subspace in a topological vector space $X$, by $X_{1}^{0}$ we denote its polar (or annihilator), i.e., the set of functionals $X^{*}$ vanishing at $X_{1}$.

Statement $(I)$ is equivalent to $\left(\operatorname{Ker} M_{G}+(\psi)\right)^{0}=\left(\operatorname{Ker} M_{G}\right)^{0} \cap((\psi))^{0}=\{0\}$. It follows from Lemma 2 of work [13] that Statement $(I I)$ is equivalent to the closedness of space $\left(\operatorname{Ker} M_{G}\right)^{0}+$ $((\psi))^{0}$ in $P_{D}$.

Space $P_{D}$ is a module over the ring of polynomials. Thanks to duality, polar $\left(\operatorname{Ker} M_{G}\right)^{0}$ coincides with the submodule

$$
(G)_{P_{D}}=\left\{h \in P_{D}: h=G \cdot r ; r \in P_{D}\right\}
$$


We shall show in the proof of sufficiency in Lemma 4 that $(G)_{P_{D}}=(G) \cap P_{D}$, where $(G)$ is the closed ideal in $H(\mathbb{C})$ generated by function $G$. In particular, it implies that submodule $(G)_{P_{D}}$ is closed.

As it is known, $\left(M^{*}\right)$-space $H(D)$ is reflexive ([9], [10]), i.e., its second strongly dual space $H^{* *}(D)$ is canonically isomorphic to space $H(D)$. This is why, in view of this canonical isomorphism, the mapping $\psi \longmapsto[\psi, \cdot]$ defines an isomorphism between $\left(M^{*}\right)$-space $H(D)$ and strongly dual space $P_{D}^{*}$. Each function in $H(D)$ is one-to-one correspondence to a linear continuous functional in strongly dual space $P_{D}$.

More precisely, this mapping is understood as follows: canonical isomorphism between $H(D)$ and $H^{* *}(D)$ reads as $\psi \longmapsto \Theta \psi=F_{\psi}, F_{\psi} \in P_{D}^{*},\left\langle F_{\psi}, \varphi\right\rangle=[\psi, \varphi]=\left\langle\mathcal{L}^{-1} \varphi, \psi\right\rangle$. Here $\psi \in H(D)$, $\varphi \in P_{D}$.

Each function $\psi \in H(D), \psi \not \equiv 0$, generates the convolution operator $\widetilde{M}_{\psi}: P_{D} \longmapsto P_{D}$, $\widetilde{M}_{\psi}[\varphi](z)=\left[(\Theta \psi)_{\lambda}, S_{z}(\varphi(\lambda))\right]$, in the space of entire functions of exponential type $P_{D}$. Here $S_{z}$ is a shift operator, $S_{z}(\varphi(\lambda))=\varphi(\lambda+z), \lambda \in \mathbb{C}$.

Hence, we obtain

$$
\widetilde{M}_{\psi}[\varphi](z)=\left\langle\left(\mathcal{L}^{-1} S_{z} \varphi\right)_{\lambda}, \psi(\lambda)\right\rangle=\left\langle e^{z \lambda}\left(\mathcal{L}^{-1} \varphi\right)_{\lambda}, \psi(\lambda)\right\rangle=\left\langle\left(\mathcal{L}^{-1} \varphi\right)_{\lambda}, e^{z \lambda} \psi(\lambda)\right\rangle, \quad \varphi \in P_{D}
$$

We note that employing the known formula for the inverse Borel transformation [14], one can obtain the integral representation for this operator [5], 4.

It is known that $\widetilde{M}_{\psi}$ is a linear continuous surjective operator. Operator $\widetilde{M}_{\psi}$ is adjoint to operator $\widetilde{A}_{\psi}$ of multiplication by function $\psi$ in space $H(D)$ acting on functions $g \in H(D)$ as follows: $g \longmapsto \psi \cdot g$. Operator $\widetilde{A}_{\psi}$ is linear and continuous, and its range coincides with closed ideal $(\psi)$. We denote $\operatorname{Ker} \widetilde{M}_{\psi}=\left\{f \in P_{D}: \widetilde{M}_{\psi}[f]=0\right\}$.

In view of duality, polar $((\psi))^{0}$ coincides with $\operatorname{Ker} \widetilde{M}_{\psi}$.

In the beginning of this section there has been described the scheme reducing the proof of representation (2) to Statements $(I)$ and $(I I)$. Then the following proposition has been proven.

Proposition 1. In $\left(M^{*}\right)$-space $H(D)$, Statements $(I)$ and $(I I)$ are equivalent to two dual statements in $\left(L N^{*}\right)$-space $P_{D}$, respectively:

$\left(I^{*}\right)$ The identity $(G)_{P_{D}} \cap \operatorname{Ker} \widetilde{M}_{\psi}=\{0\}$ holds true.

$\left(I I^{*}\right)$ Subspace $(G)_{P_{D}}+\operatorname{Ker} \widetilde{M}_{\psi}$ is closed in space $P_{D}$.

\section{AuXILIARY RESUlts}

We begin with a simple but important statement.

Proposition 2. Let $D_{1}$ be a domain such that $D \subset D_{1}$ and these domains have common parts of the boundaries at which all the accumulation points of set $\mathcal{M}$ are located. If under some conditions for $\Lambda$ representation (2) for space $H\left(D_{1}\right)$ with set of nodes $\mathcal{M}$ holds true, the same representations holds also for $H(D)$ with the same set of nodes.

Proof. For each function $g \in H(D)$ there exists $g_{1} \in H\left(D_{1}\right), g \cong g_{1}$ on $\mathcal{M}$. Then $g=g_{1}+\left(g-g_{1}\right)$ in domain $D$. By the hypothesis there exists $f_{1} \in \Sigma\left(\Lambda, D_{1}\right) \subset \Sigma(\Lambda, D)$ such that $\left.f_{1} \cong g_{1}\right|_{\mathcal{M}}$. In domain $D$ we obtain the representation $g=f_{1}+\left(g_{1}-f_{1}\right)+\left(g-g_{1}\right)$. The functions in the brackets belong to $H(D)$ and vanish on $\mathcal{M}$ counting the multiplicities. The proof is complete.

In what follows we shall need some properties of the polynomials of exponentials with real exponents. Such polynomials were studied in monograph [15]. 
We consider an arbitrary polynomial of exponentials

$$
p(z)=\sum_{k=0}^{s} a_{k}(z) e^{\omega_{k} z}, \quad \omega_{0}<\omega_{1}<\cdots<\omega_{s},
$$

where $a_{k}(z)$ are some polynomials and let $a_{0} \cdot a_{s} \not \equiv 0$.

By Theorem 12.9 in monograph [15] it is easy to obtain the following lemma.

Lemma 1. There exists $c_{1}>0$ such that in the exterior of the circle $\left\{z \in \mathbb{C}:|z| \geqslant c_{1}\right\}$ we have: there exist positive constants $c_{2}, c_{3}$ and two real numbers $m_{0}, m_{s}$ obeying $m_{0}>m_{s}$ or $m_{0}=m_{s}=0$ such that

$$
|p(z)| \geqslant c_{2} e^{\omega_{0} \operatorname{Re} z}
$$

for each $z$ in domain $U_{0}=\left\{z \in \mathbb{C}: \operatorname{Re}\left(z+m_{0} \ln z\right)<-c_{3}\right\}$, and

$$
|p(z)| \geqslant c_{2} e^{\omega_{s} \operatorname{Re} z}
$$

for each $z$ in domain $U_{s}=\left\{z \in \mathbb{C}: \operatorname{Re}\left(z+m_{s} \ln z\right)>c_{3}\right\}$.

For each fixed $c \in \mathbb{R}$ we consider the curve $\operatorname{Re}(z+m \ln z)=c, m \neq 0$. It is symmetric w.r.t. the real axis. For $m>0$ this curve lies in the half-plane $\operatorname{Re} z<A, A>0$, and for $m<0$ is lies in the half-plane $\operatorname{Re} z>-A, A>0$. If a point $z=x+i y$ lies on the curve, then $\left|\frac{y}{x}\right| \rightarrow \infty$, $\arg z \rightarrow \frac{\pi}{2},|z|=|y|(1+o(1))$, as $|z| \rightarrow \infty$. The considered curve approaches asymptotically an exponential curve $x+m \ln |y|=c$.

We fix $\beta \in\left[0, \frac{\pi}{2}\right)$ and for $\alpha \in\left[0, \frac{\pi}{2}-\beta\right)$ we denote $A_{\alpha}(\beta)=\{z \in \mathbb{C}:|\arg z-\beta| \leqslant \alpha\}$.

Lemma 2. Let $\omega_{s}<0$. For an arbitrary polynomial of exponentials $p$ satisfying (4) there exists $r=r(p)>0$ such that for each $z,|z|>r$, the estimate

$$
|p(z)| \geqslant c_{3} e^{\omega_{s} \cos (\beta+\alpha)|z|}
$$

holds true in angle $A_{\alpha}(\beta)$.

Proof. It is easy to see that all points $z$ in angle $A_{\alpha}(\beta)$ located outside some circle lie in domain $U_{s}$. Hence, estimate (6) for a polynomial of exponentials $p$ in domain $U_{s}$ for $|z|>c_{1}$ implies the estimate outside some circle $|z|>r$ in angle $A_{\alpha}(\beta)$. Inequality (7) follows from (6): if $z=|z| e^{i \varphi}$, then in this angle $0>\omega_{s} \operatorname{Re} e^{i \varphi} \geqslant \omega_{s} \cos (\beta+\alpha)$. The proof is complete.

Suppose that some convex domain $D$ contains all the exponents $\omega_{k}, k=0,1, \ldots, s$, for polynomial $p$ of exponentials satisfying (4). Then it is easy to show that $p \in P_{D}$.

The next lemma was proven in [4] in a slightly different formulation. We consider an arbitrary sequence of complex numbers $\mathcal{V}=\left\{v_{j}\right\}$ satisfying $\operatorname{Re} v_{j}>0$ and being discrete in a domain of complex plane. We assume that

$$
\limsup _{j \rightarrow \infty} \frac{\operatorname{Re} v_{j}}{\ln \left|v_{j}\right|}=\infty
$$

For further purposes it is important to note that if sequence $\mathcal{V}$ lies in angle $A_{\alpha}(\beta)$, condition (8) holds true.

We denote by $I_{\mathcal{V}}=\left\{f \in H(\mathbb{C}): f\left(v_{j}\right)=0, j \in \mathbb{N}\right\}$ a closed ideal in $H(\mathbb{C})($ cf. $(3))$.

Lemma 3. In the above described situation, if condition (8) holds true for $\mathcal{V}$, none of polynomials of exponentials $p \not \equiv 0$ satisfying (4) can be contained in ideal $I_{\mathcal{V}}$.

The matter is that the condition (8) for the zeroes of the ideal contradicts estimate (6). 


\section{MAIN RESUltS}

For set $\Lambda$ we introduce set $P(\Lambda)$ of limiting directions at infinity as the set of points $s \in \mathbb{S}$, for which there exists a sequence $\left\{\lambda_{n_{k}}\right\}_{k \in \mathbb{N}}$ satisfying $\lim _{k \rightarrow \infty} \lambda_{n_{k}} /\left|\lambda_{n_{k}}\right|=s, \lim _{k \rightarrow \infty}\left|\lambda_{n_{k}}\right|=\infty$. Set $P(\Lambda)$ is closed.

Analogues of the following notion often appear in the complex analysis under various names, for instance, in studying the phenomenon of analytic continuation of sum of series of exponentials, their analogues, as well as in studying elements of invariant subspaces [2, [16], [17, [6]. For further purposes we reproduce some definitions and results from work [2].

We denote $\mathbb{S}=\{s \in \mathbb{C}:|s|=1\}$. Let $S$ be a closed subset $\mathbb{S}$ and $D$ be a domain in $\mathbb{C}$. We denote $h(\varphi)=\sup _{\sigma \in D} \operatorname{Re}\left(e^{i \varphi} \sigma\right)$. If $k(\varphi): \mathbb{C} \mapsto(-\infty,+\infty]$ is the support function (in the sense of $\mathbb{R}^{2}$ ) of a convex domain $D$, then $h(\varphi)=k(-\varphi)$.

It is easy to see that $h(\varphi)$ is the support function (in the sense of $\mathbb{R}^{2}$ ) for the domain complex conjugate with $D$. Function $H(z)=\sup _{\sigma \in D} \operatorname{Re}(z \sigma)=h(\varphi)|z|, z=|z| e^{i \varphi} \in \mathbb{C}$ is positive homogeneous, lower-semicontinuous, and convex. It implies easily that function $h(\varphi)$ is lower-semicontinuous on $\mathbb{S}$.

For $s \in \mathbb{S}, s=e^{i \varphi}$, we introduce the function $d(s)=k(-\varphi), d: \mathbb{S} \mapsto(-\infty,+\infty]$. By the definition, for each $s \in \mathbb{S}, d(s)$ is the supremum of the projections of points in $D$ on the direction $\bar{s}=e^{-i \varphi}$.

For instance, given $t \in \mathbb{S}$ and $c \in \mathbb{R}$, we denote by $\Pi_{c}(\bar{t})=\{z \in \mathbb{C}: \operatorname{Re}(t z)<c\}$ the half-plane with the direction $\bar{t}$ of the outward normal to the boundary, point $z=c \bar{t}$ lies on its boundary. For $D=\Pi_{c}(\bar{t})$ we have $d(s)=+\infty, s \neq t$, and $d(s)=c, s=t$.

The set

$$
D_{S}=\left\{z \in \mathbb{C}: \operatorname{Re}(s z)<d(s), s=e^{i \varphi} \in S,\right\}
$$

is called $S$-convex hull of domain $D$.

By the definition, $S$-convex hull $D_{S}$ of each domain $D$ is the intersection, over all $s=e^{i \varphi} \in S$, of sets $\Pi(\bar{s}, D)=\{z \in \mathbb{C}: \operatorname{Re}(s z)<d(s)\}$. If there exists $t \in S: d(t)=\infty$, then $\Pi(\bar{t}, D)=\mathbb{C}$. If at that there exists at least one number $s \in S$ for which $d(s)<\infty$, in the definition of $D_{S}$ such $t \in S$ can be neglected.

If $d(s)<\infty$, set $\Pi(\bar{s}, D)$ is the support half-plane of domain $D$, i.e., $D \subset \Pi(\bar{s}, D)$ and $\partial D \cap \partial \Pi(\bar{s}, D) \neq \emptyset$. It is easy to see that $\Pi(\bar{s}, D)=\Pi_{0}(\bar{s})+\bar{s} d(s)$. Here $\Pi_{0}(\bar{s})=\{z \in \mathbb{C}$ : $\operatorname{Re}(s z)<0\})$.

Set $D_{S}$ is a convex domain, moreover, it is $S$-convex [2], [16]. If $S=\mathbb{S}, S$-convex hull of a set is its usual convex hull.

Proposition A. Let $D$ be a convex domain and $S=P(\Lambda)$. If the series of exponentials $\sum_{n=1}^{\infty} c_{n} e^{\lambda_{n} z}$ converges absolutely for each $z \in D$, it converges absolutely for $z \in D_{P(\Lambda)}$. Its sum is an analytic function in convex domain $D_{P(\Lambda)}$.

The first statement follows from Propositions 16 and 8 in work [2]. It was proven in work [1] that a series converging absolutely in a convex domain $D$ converges also in the topology of space $H(D)$ of uniform convergence on compact sets.

Domain $D$ is a half-plane. We fix $\beta,|\arg \beta|<\frac{\pi}{2}$ and we denote $s_{\beta}=e^{i \beta}$. We consider the case when $D=\Pi_{0}\left(e^{-i \beta}\right)$ is the "left" half-plane.

Suppose that in domain $D$ we are given an arbitrary infinite discrete set of real interpolation nodes $\mathcal{M} \subset \Pi_{0}\left(\overline{s_{\beta}}\right) \cap \mathbb{R}^{-}$. Each point $\mu_{k} \in \mathcal{M}$ has multiplicity $m_{k}, m_{k} \in \mathbb{N}$.

Lemma 4. Suppose that set $\mathcal{M}$ has the unique accumulation point $z=0$. In space $H\left(\Pi_{0}\left(\overline{s_{\beta}}\right)\right)$, the problem of multiple interpolation by series of exponentials in $\Sigma\left(\Lambda, \Pi_{0}\left(\overline{s_{\beta}}\right)\right)$ with set of nodes $\mathcal{M}$ is solvable if and only if $s_{\beta} \in P(\Lambda)$. 
We observe that direction $s_{\beta}$ is complex conjugate for the direction $\overline{s_{\beta}}=e^{-i \beta}$ of the outward normal to boundary $\partial \prod_{0}\left(\overline{s_{\beta}}\right)$.

Proof. By the symmetry arguments (consider functions $\overline{f(\bar{z})}$ ) in the proof we can assume that $\beta \in\left[0, \frac{\pi}{2}\right)$. The hypothesis of the lemma means that sets $\Lambda \cap A_{\alpha}(\beta)$ are infinite for all sufficiently small $\alpha$.

Necessity. Suppose that the problem of multiple interpolation by series of exponentials in $\Sigma\left(\Lambda, \Pi_{0}\left(\overline{s_{\beta}}\right)\right)$ with set of nodes $\mathcal{M}$ is solvable. Assume that $s_{\beta} \notin P(\Lambda)$. Then closed set $P(\Lambda)$ is separated from direction $s_{\beta}$ conjugate to the direction $\overline{s_{\beta}}$ of the outward normal to boundary $\partial \Pi_{0}\left(\overline{s_{\beta}}\right)$.

For each $s=e^{i \varphi} \in P(\Lambda)$ we have $s \neq s_{\beta}$. Hence, for the domain $D=\Pi_{0}\left(\overline{s_{\beta}}\right)$ the identity $d(s)=+\infty$ holds true. Therefore, for each $s \in P(\Lambda)$ we get $\Pi(\bar{s}, D)=\mathbb{C}$. And by the definition of $S$-convex hull we obtain $D_{P(\Lambda)}=\mathbb{C}$.

Proposition A implies the following fact. If a series of exponentials converges absolutely in $\Pi_{0}\left(\overline{s_{\beta}}\right)$ and $s_{\beta} \notin P(\Lambda)$, then this series converges absolutely in $\mathbb{C}$ and its sum is an entire function.

Interpolation by entire functions with arbitrary (for instance, unbounded) data on the set of nodes $\mathcal{M}$ with a finite accumulation point is impossible that leads us to the contradiction.

Sufficiency. The proof consists of two steps.

1. First we reduce the problem to the interpolation in the kernel of convolution operator. If the statement of the lemma is proven for $\widetilde{\Lambda} \subset \Lambda$, it will be true also for $\Lambda$. In what follows, we pass to a special subspace in $\Sigma(\Lambda, D)$ closed in $H(D)$. In order to do it, we replace the set of exponents by a sequence in $\Lambda$.

Passing to a subsequence we can assume that

1. $\Lambda \subset A_{\alpha}(\beta)$ for some small $\alpha$.

2. $P(\Lambda)=\left\{s_{\beta}\right\}$.

3. The separation condition

$$
\left|\lambda_{n+1}\right|>2\left|\lambda_{n}\right|
$$

holds true.

By $G$ we denote an entire function with simple zeroes $\lambda_{n}$,

$$
G(z)=\prod_{n=1}^{\infty}\left(1-\frac{z}{\lambda_{n}}\right) .
$$

The quantity $\delta=\lim \sup _{n \rightarrow \infty} \frac{1}{\left|\lambda_{n}\right|} \ln \frac{1}{\left|G^{\prime}\left(\lambda_{n}\right)\right|}$ is Gelfand-Leont'ev index.

It follows from (9) that function $G$ has the minimal type at order 1 and the condensation index is $\delta=0$. It was shown in work [4].

The results of the monograph [11, Thm. 4.2.2] implies the following statement.

Let $\delta=0$. Consider the closure in sense of the topology in $H(D)$ of a linear span of polynomial-exponential monomials with the exponents having the upper density counting multiplicities. Each function in this closure is represented by a series of exponentials.

Subspace Ker $M_{G}$ admits spectral synthesis. Then, in view of Theorem 4.2.3 in monograph [11, we obtain the following statement.

Proposition B. Kernel Ker $M_{G}$ is formed by functions $f(z)$ represented by the series of exponentials,

$$
f(z)=\sum_{n=1}^{\infty} c_{n} e^{\lambda_{n} z}, \quad z \in \mathbb{C}
$$


converging in the topology of space $H(D)$, i.e., $\operatorname{Ker} M_{G}=\Sigma(\Lambda, D)$.

It should be noticed that in the multi-dimensional case in a more general situation of invariant subspaces, in work [18] the fundamental principle was studied (in our case it is Proposition B). The most general formulation of this problem for a complex plane was considered in [19]. In work [20] series with real exponents $\Lambda$ were studied in details.

In these works a new characteristics $S_{\Lambda}$ was introduced. Employing this characteristics, the authors succeeded to obtain the validity criterions for the fundamental principle for invariant subspaces in convex domains. By the results of the first step, $\Lambda$ is located in an angle. Then, reproducing word-by-word the proof of Lemma 1 in [20], we obtain that $S_{\Lambda}=0$ and Proposition B can be obtained by the results in [18], [19], [20].

2. At this step we proceed to proving dual statements.

We denote by $\psi$ a function in $H(D)$ with zero set $\mathcal{M}$ counting the multiplicities $m_{k}$.

By Proposition 1, the solvability of interpolation problem follows from two dual statements: $\left(I^{*}\right)$ The identity $(G)_{P_{D}} \cap \operatorname{Ker} \widetilde{M}_{\psi}=\{0\}$ holds true.

$\left(I I^{*}\right)$ Subspace $(G)_{P_{D}}+\operatorname{Ker} \widetilde{M}_{\psi}$ is closed in space $P_{D}$.

Submodule $(G)_{P_{D}}$ has been introduced in (3).

An important ingredient in the proof of Statements $\left(I^{*}\right)$ and $\left(I I^{*}\right)$ is the following known fact.

Subspace $\operatorname{Ker} \widetilde{M}_{\psi} \subset P_{D}$ is the linear span of all the monomials $\left\{z^{\nu} e^{\mu_{k} z}\right\}, k \in \mathbb{N}, \nu=$ $0,1, \ldots, m_{k}-1$, i.e., it consists of exponentials (4), where $\omega_{k}=\mu_{k}$.

This is an easily provable fundamental principle for $\operatorname{Ker} \widetilde{M}_{\psi}$ in space $P_{D}$.

Dual statement $\left(I^{*}\right)$ follows from Lemma 3: let us show that a polynomial of exponentials $p \in \operatorname{Ker} \widetilde{M}_{\psi}, p \not \equiv 0$, can not belong to $(G)_{P_{D}}$. Indeed, in view of Step 1 , we assume that set $\Lambda$ lies in $A_{\alpha}(\beta)$. It implies that sequence $v_{k}=\lambda_{k}$ satisfies condition (8) in Lemma 3. We observe that $I_{\Lambda}=(G)$ is a closed ideal in $H(\mathbb{C})$. As it was mentioned in $(3),(G)_{P_{D}}=(G) \cap P_{D}$. Statement $\left(I^{*}\right)$ is proven.

Let us prove the last identity. By the definition, $(G)_{P_{D}} \subset I_{\Lambda} \cap P_{D}$. In accordance with Theorem [14] on division by a function of minimal type in space $P_{D}$, the opposite inclusion holds true as well. In other words, it is a corollary of the theorem on summing indicators. Submodule in the right hand side of the last identity is closed since the topology in $P_{\mathbb{C}}$ is stronger than the topology of pointwise convergence. Hence, submodule $(G)_{P_{D}}$ is closed in $P_{D}$. These facts were used above in obtaining the dual formulation of interpolation problem (Proposition 1).

For the direct algebraic sum $(G)_{P_{D}} \oplus \operatorname{Ker} \widetilde{M}_{\psi}$ we obtain $(G)_{P_{D}} \oplus \operatorname{Ker} \widetilde{M}_{\psi} \subset P_{D}$. Let us prove the closedness of this subspace in $P_{D}$ (it is statement $\left(I I^{*}\right)$ ). As it is known [9], in $\left(L N^{*}\right)$-space $P_{D}$, the closedness of its arbitrary subspace $X$ is equivalent to its sequential closedness.

Convergence of a sequence $\left\{g_{l}\right\}_{l \in \mathbb{N}}$ in $\left(L N^{*}\right)$-topology of space $P_{D}$ means the following:

1. Sequence $\left\{g_{l}\right\}$ converges to $g$ in the topology of space $H(\mathbb{C})$.

2. There exist $A>0, j \in \mathbb{N}$ such that for each $l \in \mathbb{N}$ the estimate

$$
\left|g_{l}(z)\right| \leqslant A e^{H_{j}(z)}, \quad z \in \mathbb{C},
$$

\section{holds true.}

Here $\left\{K_{j}\right\}$ is an arbitrary fixed countable exhausting of domain $D$ by compact sets: $K_{j} \subset$ $\operatorname{int} K_{j+1}$ and $D=\bigcup_{j \in \mathbb{N}} K_{j}, H_{j}(z)=\sup _{\sigma \in K_{j}} \operatorname{Re} z \sigma$. If $z=|z| e^{i \varphi}, h_{j}(\varphi)=H_{j}(z) /|z|$ is the support function (in the sense of $\mathbb{R}^{2}$ ) of the compact set complex conjugate with $K_{j}$. 
We consider an arbitrary sequence $\left\{g_{l}\right\}_{l \in \mathbb{N}}$ of functions in $(G)_{P_{D}} \oplus \operatorname{Ker} \widetilde{M}_{\psi}$ and assume that it converges to a function $g \in P_{D}$ in space $P_{D}$. Let us show that limiting function $g$ belongs to $(G)_{P_{D}} \oplus \operatorname{Ker} \widetilde{M}_{\psi}$.

Sequence $\left\{g_{l}\right\}$ consists of functions $g_{l}=p_{l}+R_{l}$, where $R_{l} \in(G)_{P_{D}}$, i.e., $\left.R_{l}\right|_{\Lambda}=0$, while $p_{l} \in \operatorname{Ker} \widetilde{M}_{\psi}$. If sequence $\left\{g_{l}\right\}$ contains infinitely many terms with $R_{l} \equiv 0$, then $g \in \operatorname{Ker} \widetilde{M}_{\psi}$. If in $\left\{g_{l}\right\}$ there are infinitely many terms with $p_{l} \equiv 0$, then $g \in(G)_{P_{D}}$. For sequences $\left\{g_{l}\right\}$ of such type we have $g \in(G)_{P_{D}} \oplus \operatorname{Ker} \widetilde{M}_{\psi}$.

Therefore, in what follows we can assume that sequence $\left\{g_{l}\right\}$ is so that $R_{l} \not \equiv 0, p_{l} \not \equiv 0$ for each $l$.

We suppose that $\mu_{k}<\mu_{k+1}<0, \mu_{k} \rightarrow 0, k \rightarrow \infty$. Since $p_{l} \in \operatorname{Ker} \widetilde{M}_{\psi}, p \not \equiv 0$, this is a polynomial of exponentials

$$
p_{l}(z)=\sum_{\operatorname{Fin}_{\mathcal{M}}^{(l)}} a_{k}^{(l)}(z) e^{\mu_{k} z} .
$$

Here for each $k \in \mathbb{N}$ functions $a_{k}^{l}$ are arbitrary polynomials of degree at most $m_{k}-1$. For each $l \in \mathbb{N}$ in the right hand side we have the sum over a finite subset $\operatorname{Fin}_{\mathcal{M}}^{(l)} \subset \mathcal{M}$. We denote by $u_{l}$ the index of maximal $\mu_{k}$ in this representation, i.e., $a_{u_{l}}^{l} \not \equiv 0$.

Suppose that sequence $\left\{g_{l}\right\}$ is such that the set of numbers $\left\{u_{l}\right\}$ is infinite. Let us show that it is bounded. Assume that set $\left\{u_{l}\right\}$ is unbounded.

We exhaust half-plane $\Pi_{0}\left(\overline{s_{\beta}}\right)$ by half-circles $K_{j}=e^{-i \beta} \cdot B_{j}^{-}$, where $B_{j}^{-}=(-1 / j+\{|z| \leqslant$ $j\}) \cap\{\operatorname{Re} z \leqslant-1 / j\}$. For each $j$ we denote $t_{j}=\operatorname{arctg} j^{2}$. We let $\varepsilon_{j}=\frac{1}{2}\left(\frac{\pi}{2}-t_{j}\right)$. It is easy to prove the estimates

$$
-\frac{1}{j}|z| \leqslant H_{j}(z) \leqslant-A_{j}|z| \text { for } z \in A_{\varepsilon_{j}}(\beta)
$$

where $A_{j}=\frac{\sqrt{1+j^{4}}}{j^{2}} \sin \varepsilon_{j}=\frac{1}{2 j^{2}}(1+o(j)), j \rightarrow \infty$.

All the polynomials of exponentials $p_{l}$ satisfy (1). We choose $k>j$ such that $\varepsilon_{k}<\frac{\pi}{2}-\beta$. Since $a_{-q_{l}}^{(l)} \not \equiv 0$, we can apply estimate (7) for $p_{l}$ in Lemma 2 with $\alpha=\varepsilon_{k}$. Employing also estimate (10), we get the following inequality for $R_{l}=g_{l}-p_{l}, p_{l} \neq \equiv 0, R_{l} \neq \equiv 0$ :

$$
\left|R_{l}(z)\right| \geqslant\left|p_{l}(z)\right|-\left|g_{l}(z)\right| \geqslant c_{3} e^{\mu_{u_{l}} \cos (\beta+\alpha)|z|}-A e^{H_{j}(z)},
$$

for each $z$ in domain $\left\{z \in A_{\varepsilon_{k}}(\beta),|z|>r\right\}$, where $r=r(l)$. Since $k>j, A_{\varepsilon_{k}}(\beta) \subset A_{\varepsilon_{j}}(\beta)$, by (11) we obtain that

$$
\left|R_{l}(z)\right| \geqslant\left|p_{l}(z)\right|-\left|g_{l}(z)\right| \geqslant c_{3} e^{\mu_{u_{l}} \cos (\beta+\alpha)|z|}-A e^{-A_{j}|z|},
$$

outside some circle $|z|>r$ in angle $A_{\varepsilon_{k}}(\beta)$.

By assumption, set $u_{l}$ is unbounded, thus, in the representations of polynomials of exponentials $p_{l}$ there exist $\mu_{u_{l}}$ arbitrarily close to 0 .

We choose $\mu_{u_{l_{0}}}>A_{j} / \cos (\beta+\alpha)$. It implies that $\left|R_{l_{0}}(z)\right|>0$ for each $z$ outside some circle $\left\{|z|>r_{1}\left(l_{0}\right)\right\}$ in angle $A_{\varepsilon_{k}}(\beta)$.

We obtain the contradiction: indeed, by Step $1, P(\Lambda)=\left\{s_{\beta}\right\}$, this is why for each $k$ outside any circle in angle $A_{\varepsilon_{k}}(\beta)$ there exists an infinite sequence of points in $\Lambda$, while we are given that $\left.R_{l_{0}}\right|_{\Lambda}=0$.

It should be notices for arbitrary sequence $\left\{g_{l}\right\}$ compact set $K_{j}$ can be arbitrarily large while quantity $\varepsilon_{k}$ can be arbitrarily small as the assumption of the lemma says.

Hence, in the representations for polynomials of exponentials $p_{l}$ in an arbitrary converging sequence $\left\{g_{l}\right\}, g_{l}=p_{l}+R_{l}$, set of numbers $u_{l}$ is bounded. Therefore, sequence $\left\{p_{l}\right\}$ belongs to 
some finite-dimensional subspace $X \subset \operatorname{Ker} \widetilde{M}_{\psi_{1}}$. Statement $\left(I^{*}\right)$ means that all the elements of converging sequence $g_{l}=p_{l}+R_{l}$ lies in the algebraic direct sum $X \oplus(G)_{P_{D}} \subset \operatorname{Ker} \widetilde{M}_{\psi_{1}} \oplus(G)_{P_{D}}$.

In each topological vector space algebraic sum of a finite-dimensional subspace and a closed subspace is a closed subspace [21]. Thus, limiting function $g$ of sequence $g_{l}=p_{l}+R_{l}$ belongs to $\operatorname{Ker} \widetilde{M}_{\psi} \oplus(G)_{P_{D}}$. Statement $\left(I I^{*}\right)$ is proven.

Proven statements $\left(I^{*}\right)$ and $\left(I I^{*}\right)$ complete the proof of Lemma 4.

Remark 1. In the proof of sufficiency we have shown the following fact. Let $\Lambda$ be an arbitrary set of exponents. Then condition (8) (implied by the hypothesis of Lemma 4) is sufficient for set $\Sigma\left(\Lambda, \Pi_{0}\left(\overline{s_{\beta}}\right)\right)+I_{\mathcal{M}}$ to be everywhere dense in the topology of space $H\left(\Pi_{0}\left(\overline{s_{\beta}}\right)\right)$.

Remark 2. By the transformation $z \rightarrow-z$ of plane $\mathbb{C}$ we obtain the formulation corresponding to the "right" half-plane. Moreover, in the considered problem for each $h \in \mathbb{R}$ the transformation $z \rightarrow z+h$ of the complex plane is admissible after which we need to change the formulations in an appropriate way. Indeed, under such transformation the set of series of exponentials is preserved while the set of nodes is shifted.

\section{Domain $D$ is convex with an accumulation point $\mathcal{M}$ at the boundary.}

Let $D$ be a convex domain in $\mathbb{C}$. We denote $h(\varphi)=\sup _{\sigma \in D} \operatorname{Re}\left(e^{i \varphi} \sigma\right)$. For each $\varphi$, number $h(\varphi)$ is the value of the support function $k(-\varphi)$ (in the sense of $\mathbb{R}^{2}$ ) for domain $D$ in the direction $e^{-i \varphi}$. Let $s=e^{i \varphi}$; function $d(s)=k(-\varphi)$ was introduced above.

The straight line $l(\bar{s})=\{z=x+i y: \operatorname{Re}(s z)=x \cos (-\varphi)+y \sin (-\varphi)=d\}$ is called the support one for domain $D$ in the direction $\bar{s}=e^{-i \varphi}$, if at the boundary of $D$ there exists a point belonging to $l(\bar{s})$ and domain $D$ lies in the support half-plane $\Pi(\bar{s}, D)=\{z \in \mathbb{C}: \operatorname{Re}(s z)<d\}$. We call this point the support point for line $l(\bar{s})$. It is easy to see that line $l(\bar{s})$ is support if and only if $d=d(s)$.

Let $0 \in \partial D$. We denote by $T_{D}(0) \subset \mathbb{S}$ the set of all $s \in \mathbb{S}$ for which point 0 at boundary $D$ is a support one for $l(\bar{s})$. It is clear that $T_{D}(0)=\{s \in \mathbb{S}: d(s)=0\}$.

We observe that under the hypothesis of Lemma $4, D=\Pi_{0}\left(\overline{s_{\beta}}\right), T_{D}(0)=\left\{s_{\beta}\right\}$.

Theorem 1. Let $D$ be a convex domain, $0 \in \partial D$ and $D \cap \mathbb{R} \neq \emptyset$. Suppose that set $\mathcal{M} \subset D \cap \mathbb{R}$ is discrete in $D$ and has the unique accumulation point. Problem of multiple interpolation by the series of exponentials in $\Sigma(\Lambda, D)$ with set of nodes $\mathcal{M}$ is solvable if and only if $P(\Lambda) \cap T_{D}(0) \neq \emptyset$.

Proof. The case $D=\Pi_{0}\left(\overline{s_{\beta}}\right),|\beta|<\frac{\pi}{2}$, was considered in Lemma 4.

Without loss of generality we can assume that $D \cap \mathbb{R}^{-} \neq \emptyset$, then $\mathcal{M} \subset D \cap \mathbb{R}^{-}$; otherwise we can employ transformation $z \rightarrow-z$ of plane $\mathbb{C}$.

Then $h(\varphi) \geqslant 0$ and the lower semi-continuity yield that set $T_{D}(0)$ is closed. The convexity and homogeneity of function $H(z)=h(\varphi)|z|$ imply that $T_{D}(0)$ is a connected set.

It is also easy to see that $|\arg s|<\frac{\pi}{2}$ for each $s \in T_{D}(0)$ since $\mathcal{M} \cap \mathbb{R}^{-} \neq \emptyset$.

The necessity of condition $P(\Lambda) \cap T_{D}(0) \neq \emptyset$ follows from Proposition B, while the sufficiency is proven by reducing to Lemma 4 .

Necessity. Suppose that the interpolation problem is solvable but the hypothesis of the theorem is not true, $P(\Lambda) \cap T_{D}(0)=\emptyset$. In what follows we shall show that in this case the point $z=0$ lies in $D_{P(\Lambda)}$.

Sets $P(\Lambda)$ and $T_{D}(0)=\{s \in \mathbb{S}: d(s)=0\}$ are closed.

For each subset $X \subset \mathbb{S}$ and number $\delta>0$ we denote

$$
X_{\delta}=\{s \in \mathbb{S}: \exists u \in X,|s-u| \leqslant \delta\} .
$$

There exists $\delta>0$ such that $P(\Lambda) \cap\left(\left(T_{D}(0)\right)_{\delta}=\emptyset\right.$. Hence, there exists a connected closed set $S_{1} \in \mathbb{S}$ such that $P(\Lambda) \in \operatorname{int} S_{1}, S_{1} \cap T_{D}(0)=\emptyset$. 
The definition of $S$-convex hull implies that

$$
D_{S_{1}} \subset D_{P(\Lambda)} \text {. }
$$

For each $s \in S_{1}$ we have $d(s)>0$. Hence, the semi-continuity yields that $\exists c, d(s)>c>0$, $s \in S_{1}$. We denote $B(c)=\{z \in \mathbb{C}:|z|=c\}$. For each $z \in B(c)$ and each $s \in S_{1}$ we have $\operatorname{Re}(s z)<c<d(s)$. It follows that point $0 \in \partial D$ lies in $\Pi_{c}(\bar{s}) \subset \Pi(\bar{s}, D)$ for each $s \in S_{1}$. It is proven that $0 \in(B(c))_{S_{1}} \subset D_{S_{1}}$.

By (12) we obtain that $0 \in D_{P(\Lambda)}$. We complete the proof as follows.

By Proposition A, each series of exponentials converging absolutely in convex domain $D$ converges absolutely in convex domain $D_{P(\Lambda)}$. Its sum is an analytic function in $D_{P(\Lambda)}$.

Point $z=0$ lies in domain $D_{P(\Lambda)}$ and by the assumption, it is the accumulation one for set of nodes $\mathcal{M}$. For set of nodes $\mathcal{M}$ having an accumulation point in the domain, the interpolation by analytic in this domain functions is impossible for arbitrary (for instance, unbounded) interpolation data and it leads us to the contradiction. The proof of the necessity is complete.

Sufficiency. By the assumption, there exists a limiting direction $s_{\beta}=e^{i \beta} \in P(\Lambda)$ lying in $T_{D}(0)$. Hence, $|\beta|<\frac{\pi}{2}$ since the set $D \cap \mathbb{R}^{-}$is non-empty.

Since $s_{\beta} \in T_{D}(0)$, point $z=0$ is the support one for the straight line $l\left(\overline{s_{\beta}}\right)=\left\{z: \operatorname{Re}\left(z s_{\beta}\right)=\right.$ $0\}$. Domain $D$ lies in the support half-plane $\Pi_{0}\left(\overline{s_{\beta}}\right)=\left\{\operatorname{Re}\left(z s_{\beta}\right)<0\right\}$ and $0 \in \partial \Pi_{0}\left(\overline{s_{\beta}}\right)=l\left(\overline{s_{\beta}}\right)$. Thus, set $\mathcal{M} \subset \Pi_{0}\left(\overline{s_{\beta}}\right)$ can be employed as the set of nodes for interpolation by series of exponentials in space $H\left(\Pi_{0}\left(\overline{s_{\beta}}\right)\right) \subset H(D)$. Under such conditions, the solvability for problem of interpolation by series of exponentials in space $H\left(\Pi_{0}\left(\overline{s_{\beta}}\right)\right)$ was proven in Lemma 4 . The solvability of the problem in $H(D)$ follows from Proposition 2. The proof is complete.

The authors express their gratitude to the participants of Ufa city seminar on theory of functions for the attention to the work and a useful discussion.

\section{BIBLIOGRAPHY}

1. A.F. Leont'ev. Entire functions. Exponential series. Nauka, Moscow (1983). (in Russian).

2. S.G. Merzlyakov. Integrals of exponential Radon measures // Ufimskij Matem. Zhurn. 3:2, 57-80 (2011). [Ufa Math. J. 3:2, 56-78 (2011).]

3. L. Hörmander. An introduction to complex analysis in several variables. D. van Nostrand Company, Princeton (1966).

4. S.G. Merzlyakov, S.V. Popenov. Interpolation with multiplicity by series of exponentials in $H(\mathbb{C})$ with nodes on the real axis //Ufimskij Matem. Zhurn. 5:3, 130-143 (2013). [Ufa Math. J. 5:3, 127-140 (2013).]

5. V.V. Napalkov, A.A. Nuyatov. The multipoint de la Vallée-Poussin problem for a convolution operator // Matem. Sbornik. 203:2, 77-86 (2012). [Sb. Math. 203:2, 224-233 (2012).]

6. A.S. Krivosheev. A criterion for analytic continuation of functions from invariant subspaces in convex domains of the complex plane // Izv. RAN. Ser. Matem. 68:1, 43-78 (2004). [Izv. Math. 68:1, 43-76 (2004).]

7. K.R. Zimens, V.V. Napalkov. The multiple de la Vallée-Poussin problem on convex domains in the kernel of the convolution operator // Dokl. RAN. 458:4, 387-389 (2014). [Dokl. Math. 89:3, 287-289 (2014).]

8. V.V. Napalkov, S.V. Popenov. The holomorphic Cauchy problem for the convolution operator in analytically uniform spaces, and Fisher expansions // Dokl. RAN. 381:2, 164-166 (2001). [Dokl. Math. 64:3, 330-332 (2001).]

9. J. Sebastian-e-Silva. "On some classes of locally convex spaces important in applications" // Matematika. Sbornik Perevodov. 1, 60-77 (1957). (in Russian).

10. V.V. Napalkov. Convolution equations in multidimensional spaces. Nauka, Moscow (1982). (in Russian). 
11. A.F. Leont'ev. Sequences of exponential polynomials. Nauka, Moscow (1980). (in Russian).

12. I.F. Krasičkov-Ternovskiǐ. Invariant subspaces of analytic functions. iii. on the extension of spectral synthesis. Matem. Sbornik. 88(130):1(5), 3-30 (1972). [Math. USSR. Sb. 17:3, 327-348 (1972).]

13. S.G. Merzlyakov. Invariant subspaces of the operator of multiple differentiation // Matem. Zametki. 33:5, 701-713 (1983). [Math. Notes Acad. Sci. USSR. 33:5, 361-368 (1983).]

14. A.F. Leontiev. Exponential series. Nauka, Moscow (1976). (in Russian).

15. R. Bellman, K.L. Cooke. Differential-difference equations. Math. Sci. Eng. 6. Academic Press, New York (1963).

16. O.A. Krivosheeva. The convergence domain for series of exponential monomials // Ufimskij Matem. Zhurn. 3:2, 43-56 (2011). [Ufa Math. J. 3:2, 42-55 (2011).]

17. O.A. Krivosheeva. Convergence domain for series of exponential polynomials // Ufimskij Matem. Zhurn. 5:4, 84-90 (2013). [Ufa Math. J. 5:4, 84-90 (2013).]

18. A.S. Krivosheev. A fundamental principle for invariant subspaces in convex domains // Izv. RAN. Ser. Matem. 68:2, 71-136 (2004). [Izv. Math. 68:2, 291-353 (2004).]

19. O.A. Krivosheeva, A.S. Krivosheev. A criterion for the fundamental principle to hold for invariant subspaces on bounded convex domains in the complex plane // Funkts. Anal. Pril. 46:4, 14-30 (2012). [Funct. Anal. Appl. 46:4, 249-261 (2012).]

20. A.S. Krivosheev, O.A. Krivosheeva. A closedness of set of Dirichlet series sum // Ufimskij Matem. Zhurn. 5:3, 96-120 (2013). [Ufa Math. J. 5:3, 94-117 (2013).]

21. W. Rudin. Functional analysis. McGraw-Hill Book Company, New York (1973).

Sergei Georgievich Merzlyakov,

Institute of Mathematics CC USC RAS

Chernyshevsky str., 112,

450008, Ufa, Russia

E-mail: msg2000@mail.ru

Sergei Viktorovich Popenov,

Institute of Mathematics CC USC RAS

Chernyshevsky str., 112,

450008, Ufa, Russia

E-mail: spopenov@gmail.com 